\title{
Acurácia de pontos de corte de IMC e circunferência da cintura para a predição de obesidade em idosos
}

\author{
Accuracy of BMI and waist circumference cut-off points to predict \\ obesity in older adults
}

Erika Aparecida Silveira (https://orcid.org/0000-0002-8839-4520) ${ }^{1}$

Valéria Pagotto (https://orcid.org/0000-0002-5590-2453) ${ }^{2}$

Larissa Silva Barbosa (https://orcid.org/0000-0002-2564-6524) ${ }^{1}$

César de Oliveira (https://orcid.org/0000-0002-4099-4762) ${ }^{3}$

Georgia das Graças Pena (https://orcid.org/0000-0002-0360-223X) ${ }^{4}$

Gustavo Velasquez-Melendez (https://orcid.org/0000-0001-8349-5042) ${ }^{5}$
${ }^{1}$ Faculdade de Medicina, Universidade Federal de Goiás. Av. Primeira Avenida $\mathrm{s} / \mathrm{n}^{\circ}$, Setor Leste Universitário. 74000000 Goiânia GO Brasil. erikasil@terra.com.br

${ }^{2}$ Faculdade de Enfermagem, Universidade Federal de Goiânia. Goiânia GO Brasil.

${ }^{3}$ Department of Epidemiology and Public Health, University College. London UK.

${ }^{4}$ Faculdade de Medicina,

Universidade Federal de

Uberlândia. Uberlândia

MG Brasil.

${ }^{5}$ Escola de Enfermagem,

Universidade Federal

de Minas Gerais. Belo

Horizonte MG Brasil.

\begin{abstract}
The main objectives were to analyse the validity and accuracy of Body Mass Index (BMI) and Waist Circumference (WC) to evaluate obesity by excess of body fat in older adults and to identify more adequate cut-off points for this age group. The recommended cut-off points for BMI $\left(25,27\right.$ or $\left.30 \mathrm{~kg} / \mathrm{m}^{2}\right)$ and $W C(\geq 102 \mathrm{~cm}$ for men and $\geq 88 \mathrm{~cm}$ for women or $\geq 90 \mathrm{~cm}$ for men and $\geq 80 \mathrm{~cm}$ for women) were compared to the total body densitometry. BF was defined by a value higher than the 90th percentile. Out of the 132 participants, $61 \%$ were women and aged between 60 and 91 years. The recommended cut-off points of $B M I \geq 25 \mathrm{~kg} / \mathrm{m}^{2}$ and $B M I \geq 27 \mathrm{~kg} / \mathrm{m}^{2}$ showed similar performances. BMI $\geq 30 \mathrm{~kg} / \mathrm{m}^{2}$ showed high specificity but low sensitivity to identify $B F$ in men and better performance in women. Conventional WC cut-off points showed low sensitivity and specificity. Based on our analyses, new cut-off points for BMI $\left(25 \mathrm{~kg} / \mathrm{m}^{2}\right.$ for men and $26.6 \mathrm{~kg} / \mathrm{m}^{2}$ for women) and WC (98.8 cm for men and $77.8 \mathrm{~cm}$ for women) were proposed. The new cut-off points showed sensitivity and specificity values $>74 \%$ and accuracy $>76 \%$. The areas under the curve (ROC) were $>0.86$. The new BMI and WC cut-off points proposed in the present study for the diagnosis of obesity in older adults showed the best levels of sensitivity and specificity for this age group. Keywords Sensitivity and Specificity, Obesity, Body composition, Ageing
\end{abstract}

Resumo O objetivo deste estudo foi analisar a validade e a acurácia do Índice de Massa Corporal (IMC) e da Circunferência da Cintura (CC) na avaliação de obesidade avaliada pelo excesso de gordura corporal (GC) e propor pontos de corte mais adequados para idosos. Os pontos propostos da literatura IMC $\left(25,27\right.$ ou $\left.30 \mathrm{~kg} / \mathrm{m}^{2}\right)$ e CC $(\geq 102 \mathrm{~cm}$ -homens $e \geq 88 \mathrm{~cm}$-mulheres ou $\geq 90 \mathrm{~cm}$-homense $\geq 80 \mathrm{~cm}$-mulheres) foram avaliados conforme densitometria corporal total. A elevada GC foi definida por percentil > 90. Dos 132 idosos, 60,6\% eram mulheres de 60-91 anos. Os pontos de corte recomendados de IMC $\geq 25 e \geq 27 \mathrm{~kg} / \mathrm{m}^{2}$ apresentaram desempenhos similares, sendo que o IMC $\geq 30 \mathrm{~kg} / \mathrm{m}^{2}$ apresentou alta especificidade e baixa sensibilidade no sexo masculino e melhor desempenho para GC nas mulheres. Os pontos de corte convencionais de CC não apresentaram boa sensibilidadelespecificidade. Foram propostos novos pontos de corte para IMC (masculino-25 kg/m²; feminino-26,6 kg/m ${ }^{2}$ ) CC (masculino-98,8 cm; feminino-77,8 cm). Estes valores demonstraram sensibilidade e especificidade $>74 \%$ e acurácia $>76 \%$. As áreas sob a curva foram $>0,86$. O presente estudo propõe a utilização de pontos de corte mais acurados para diagnóstico de obesidade/GC em idosos, sendo para homens IMC $25 \mathrm{~kg} / \mathrm{m}^{2}$ e CC de $98,8 \mathrm{~cm}$ e para mulheres IMC de $26,6 \mathrm{~kg} / \mathrm{m}^{2}$ e CC de 77,8cm, com melhores niveis de sensibilidade e especificidade.

Palavras-chave Sensibilidade e especificidade, Obesidade, Composição corporal, Envelhecimento 


\section{Introdução}

A obesidade continua sendo um dos principais problemas de saúde pública na atualidade e encontra-se em níveis pandêmicos ${ }^{1}$, sendo associada a problemas clínicos e doenças crônicas múltiplas $^{2}$. Apesar de extensivamente investigada, não há consenso sobre o melhor método para seu diagnóstico em idosos, sendo o índice de massa corporal (IMC) e a Circunferência da Cintura (CC) os mais utilizados para identificar obesidade global e central, respectivamente. Nesse sentido, estudos que confirmem ou avaliem sua capacidade preditora são necessários a fim de se obter um consenso sobre o desempenho dos pontos de corte para diagnótico em diferentes segmentos populacionais. Evidências demonstraram que em população adulta o IMC tem alta especificidade, mas baixa sensibilidade ${ }^{3}$ para identificar adiposidade $^{4}$.

Levando em consideração as mudanças na composição corporal, tais como a redistribuição da gordura corporal e redução da massa muscular e óssea no idoso, a aplicabilidade e a acurácia dos mesmos valores diagnósticos propostos para adultos em pessoas idosas pode ser inapropriado e não possui ampla aceitação ${ }^{4}$. Esses estudos são escassos na literatura, sendo que os poucos existentes analisaram a capacidade preditiva do IMC para risco cardiovascular ${ }^{5}$ e identificação da gordura corporal ${ }^{6}$, sem entretanto propor pontos de corte para essa população ${ }^{4}$. Diante do envelhecimento da população torna-se cada vez mais relevante, no campo da Saúde Coletiva e para a práticas dos profissionais nos serviços de saúde, identificar pontos de corte mais acurados para predizer a gordura corporal em idosos de forma a aprimorar o dignostico nutricional e a proposição de intervenções.

Em relação à CC, os pontos de corte para a predição de risco metabólico demonstraram diferenças entre sexos em diferentes populações ${ }^{4,7}$. Recente metanálise demonstrou aumento do risco de morte em pessoas idosas com aumento da CC mesmo entre as diferentes categorias de IMC, sugerindo a necessidade de reavaliar seus pontos de corte de CC para idosos ${ }^{8}$. Diante desse contexto, este estudo se propôs a analisar a validade e a acuracia do IMC e do CC na predição de obesidade avaliada pelo excesso de gordura coporal e identificar pontos de corte mais acurados aplicáveis a indivíduos idosos.

\section{Métodos}

O presente estudo é uma subamostra da coorte "Situação de Saúde e Indicadores Antropométricos para Avaliação do Estado Nutricional de Idosos Usuários do Sistema Único de Saúde de Goiânia", ou "Projeto Idoso/Goiânia" iniciado em 2008 com amostra probabilística de 418 idosos com 60 anos ou mais não institucionalizados, residentes na cidade de Goiania-Goiás. Foram incluídas no Projeto Idosos Goiânia pessoas com 60 anos ou mais, que foram atendidas em consulta ambulatorial no período de doze meses anteriores à coleta de dados, como forma de confirmar se eram usuários do SUS. Detalhes sobre aspectos metodológicos foram descritos em publicações prévias ${ }^{9-11}$.

O cálculo amostral para o presente estudo foi estimado considerando: nível de significância $(\alpha)$ bicaudal $=0,05, \beta=0,05$ (poder de $95 \%$ ) e coeficiente de correlação esperado de 0,35 , totalizando pelo menos 100 indivíduos ${ }^{12}$. A fim de manter a proporção de idosos de ambos os sexos bem como por categorias do índice de massa corporal (IMC), foram alocados aleatoriamente 132 idosos. Para a alocação aleatória considerou-se elegíveis aqueles que preenchiam os critérios para realização do Dual Energy X-Ray Absorptiometry - DEXA (Densitometria Corporal Total): ter peso inferior a 100kg (capacidade do equipamento) e não fazer uso de marcapasso ou algum metal no corpo, além da concordância em participar da nova etapa do estudo.

Foi realizado contato telefônico inicial e esclarecimento sobre essa etapa do estudo. Os interessados foram orientados sobre os procedimentos da entrevista e jejum para realização dos exames. No dia agendado, os idosos foram transportados até a clínica em veículo da pesquisa e as medidas realizadas por equipes previamente treinadas, $o$ que possibilitou apuração das técnicas de execução, conferindo maior acurácia das medidas.

Foram avaliados peso, altura, IMC, Circunferência da Cintura (CC) e percentual de gordura corporal (\%GC). O peso foi aferido em balança eletrônica digital, portátil com precisão de $100 \mathrm{~g}$ $\left(\right.$ Tanita $\left.^{\circ}\right)$. A estatura foi aferida utilizando-se fita métrica inextensível com precisão de $0,1 \mathrm{~cm}$ e esquadro $^{13}$.

O IMC foi calculado pela divisão do peso pelo quadrado da altura, e classificado por dois parâmetros: 1) sobrepeso, quando IMC $\geq 25 \mathrm{~kg}$ $/ \mathrm{m}^{2}$ e obesidade $\geq 30 \mathrm{Kg} / \mathrm{m}^{2}$; segundo a World Health Organization $\left.{ }^{14}, 2\right)$ excesso de peso IMC $>27 \mathrm{~kg} / \mathrm{m}^{2}$, classificação utilizada pelo Nutrition 
Screening Initiative ${ }^{15}$, recomendado também por outros autores ${ }^{16}$.

A CC foi aferida em duplicata no ponto médio entre o último arco costal e a crista ilíaca utilizando fita métrica inextensível e precisão de 0,1 $\mathrm{cm}^{17}$. Para a identificação dos pontos anatômicos das medidas antropométricas foram utilizados procedimentos padronizados ${ }^{17}$. Posteriormente, a CC foi categorizada segundo diferentes pontos de corte: 1) $\geq 94 \mathrm{~cm}$ para homens e $\geq 80 \mathrm{~cm}$ para mulheres, recomendado pela World Health Organization $^{14}$; 2) $\geq 102$ em homens e $\geq 88 \mathrm{~cm} \mathrm{em}$ mulheres recomendada pelo National Institutes of Health e pelo National Cholesterol Education Program ${ }^{18-20}$; 3) acima de 90 e $80 \mathrm{~cm}$ para homens e mulheres, respectivamente, proposto pela International Diabetes Federation ${ }^{21}$.

A gordura corporal foi avaliada em clínica especializada em exames de imagem, estimada por DEXA (Lunarò ${ }^{\circ}$ DPX-MD PLUS por meio do programa 7.52.002 DPX-L). Para o exame, os idosos usavam apenas avental, sem nenhum tipo de material metálico. $\mathrm{O}$ excesso de gordura corporal foi definido pelo percentil 90 da população estudada, estratificado por sexo e faixa etária ${ }^{22}$, seguindo os critérios previamente publicados ${ }^{23}$.

As análises estatísticas foram realizadas no STATA/SE versão 12.0. A normalidade das variáveis foi testada pelo teste Shapiro-Wilk. Para verificar diferenças de proporções por sexo usouse teste do qui-quadrado de Pearson ao nível de significância de 5\%.

Para analisar a variação da sensibilidade e especificidade de diferentes valores da Circunferência da Cintura (CC) em relação ao padrão ouro \%GC utilizou-se a Receiver Operating Characteristic (Curva ROC). Posteriormente, foram identificados os pontos de corte para CC, com respectivos valores e intervalos de confiança de sensibilidade, especificidade e acurácia. Para isso considerou-se sensibilidade e especificidade mínima de $60 \%$ e área total sob a curva ROC acima de 0,70 .

O projeto foi aprovado pelo Comitê de Ética em Pesquisa da Universidade Federal de Goiás. Todos os idosos incluídos na pesquisa foram esclarecidos em relação aos objetivos da pesquisa e assinaram o Termo de Consentimento Livre e Esclarecido.

\section{Resultados}

Foram estudados 132 idosos, com idade entre 60 e 91 anos. Em geral, foram predominantes a faixa etária de 60 a 69 anos $(52,3 \%)$ e sexo feminino $(60,6 \%)$. Os valores médios de IMC foram 25,7 \pm $4,0 \mathrm{~kg} / \mathrm{m}^{2}$ em homens e $27,4 \pm 5,7 \mathrm{~kg} / \mathrm{m}^{2}$ nas mulheres; a CC apresentou valores médios de 95,7 $\pm 11,5 \mathrm{~cm}$ e $93,7 \pm 14,5 \mathrm{~cm}$, respectivamente. $\mathrm{O}$ $\%$ GC médio foi 30,2 \pm 8,6 \% em homens e 42,8 \pm $9,0 \%$ nas mulheres. Essas variáveis apresentaram distribuição normal pelo teste de Shapiro-Wilk. As mulheres apresentaram maior frequência de obesidade global e central pelos critérios $\geq 94 \mathrm{~cm}$ para homens e $\geq 80 \mathrm{~cm}$ para mulheres (WHO) e $\geq 102$ em homens e $\geq 88 \mathrm{~cm}$ em mulheres (NCEP), bem como \%GC (Tabela 1).

Os dados da Tabela 2 mostram os desempenhos dos diferentes pontos de corte na capacidade de predição do excesso de gordura corporal. O IMC $\geq 25 \mathrm{~kg} / \mathrm{m}^{2} \mathrm{e} \geq 27 \mathrm{~kg} / \mathrm{m}^{2}$ apresentaram desempenhos semelhantes em ambos sexos, enquanto que o ponto de corte $\geq 30 \mathrm{~kg} / \mathrm{m}^{2}$ apresentou elevada especificidade porém baixa sensibilidade $(25 \%)$ no sexo masculino, predizendo melhor o elevado \%GC no sexo feminino.

A capacidade de predição da CC foi semelhante nos pontos de corte avaliados, entretanto, com valores maiores de especificidade no sexo masculino $(91,7 \%)$ e maior sensibilidade no sexo feminino $(84,4 \%)$ considerando os pontos de corte mais elevados. Quanto aos pontos de corte propostos neste estudo a partir da Curva ROC, os pontos de corte de IMC e CC com maior sensibilidade, especificidade e acurácia foram: 25,0 $\mathrm{kg} / \mathrm{m}^{2}$ e $98,8 \mathrm{~cm}$ no sexo masculino, $26,6 \mathrm{~kg} / \mathrm{m}^{2}$ e $90,5 \mathrm{~cm}$ no sexo feminino (Tabela 3 ).

Por fim, a área sob a curva (Figuras 1 e 2), mostrou que a capacidade de classificar corretamente a presença ou ausência de excesso de gordura corporal foi de $88 \%$ tanto para IMC quanto CC em homens e de $89 \%$ (IMC) e $86 \%$ (CC) nas mulheres. Todos os limites inferiores do IC 95\% foram acima de 0,78 .

\section{Discussão}

O presente estudo propõe novos pontos de corte de índice de massa corporal (IMC) e Circunferência da Cintura (CC) para diagnóstico de obesidade em idosos. Os pontos de corte mais acurados para avaliar o excesso de gordura corporal em idosos foram: IMC $\geq 25 \mathrm{~kg} / \mathrm{m}^{2}$ para homens e $\geq 26,6 \mathrm{~kg} / \mathrm{m}^{2}$ para mulheres; e CC $\geq 98,8 \mathrm{~cm}$ para homens e $\geq 90,5 \mathrm{~cm}$ para mulheres. Os resultados demonstraram que os valores das medidas antropométricas IMC e CC apresentam acurácia mais confiável (76,2 a 84,3\%) para diagnóstico de ex- 
Tabela 1. Prevalência de excesso de peso, obesidade e obesidade central por sexo, em idosos aplicando diferentes pontos de corte de IMC, CC e percentil de percentual de gordura corporal. Goiânia, Brasil (n=132).

\begin{tabular}{|c|c|c|c|c|c|}
\hline \multirow{3}{*}{ Variáveis } & \multicolumn{4}{|c|}{ Sexo } & \multirow{3}{*}{$\mathbf{p}^{\mathbf{T}}$} \\
\hline & \multicolumn{2}{|c|}{ Masculino $(n=52)$} & \multicolumn{2}{|c|}{ Feminino $(\mathbf{n}=\mathbf{8 0})$} & \\
\hline & $\mathbf{n}$ & $\%$ & $\mathbf{n}$ & $\%$ & \\
\hline \multicolumn{6}{|l|}{ Índice de Massa Corporal } \\
\hline$\geq 25 \mathrm{~kg} / \mathrm{m}^{2}$ (sobrepeso) & 31 & 59,62 & 45 & 56,25 & 0,705 \\
\hline$>27 \mathrm{~kg} / \mathrm{m}^{2}$ (excesso de peso) & 20 & 38,46 & 38 & 47,50 & 0,310 \\
\hline$\geq 30 \mathrm{~kg} / \mathrm{m}^{2}$ (obesidade) & 7 & 13,46 & 25 & 31,25 & 0,020 \\
\hline \multicolumn{6}{|l|}{ Circunferência da Cintura } \\
\hline WHO ( $\geq 94 \mathrm{~cm}$ homens $\mathrm{e} \geq 80 \mathrm{~cm}$ mulheres) & 33 & 64,71 & 65 & 81,25 & 0,033 \\
\hline $\operatorname{NCEP}(\geq 102$ homens $\mathrm{e} \geq 88 \mathrm{~cm}$ mulheres $)$ & 17 & 33,33 & 49 & 61,25 & 0,002 \\
\hline IDF ( $\geq 90$ homens e $\geq 80$ mulheres) & 38 & 74,51 & 65 & 81,25 & 0,363 \\
\hline \multicolumn{6}{|l|}{ Percentual de Gordura Corporal } \\
\hline$>$ Percentil 90 & 28 & 53,85 & 45 & 56,25 & 0,010 \\
\hline
\end{tabular}

Tabela 2. Capacidade preditiva de pontos de corte de IMC e CC anteriormente propostos na literatura para detectar gordura corporal em idosos de ambos os sexos, Goiânia, Brasil. ( $\mathrm{n}=132$ )

\begin{tabular}{|c|c|c|c|c|c|}
\hline Variáveis & $\begin{array}{c}\text { Sensibilidade } \\
\text { (IC 95\%) }\end{array}$ & $\begin{array}{c}\text { Especificidade } \\
\text { (IC 95\%) }\end{array}$ & $\begin{array}{l}\text { VPP } \\
(\%) \\
\end{array}$ & $\begin{array}{l}\text { VPN } \\
(\%)\end{array}$ & $\begin{array}{c}\text { Acurácia } \\
(\%)\end{array}$ \\
\hline \multicolumn{6}{|l|}{ Masculino $(n=52)$} \\
\hline \multicolumn{6}{|l|}{ Categorias de IMC } \\
\hline Sobrepeso $\left(\geq 25 \mathrm{~kg} / \mathrm{m}^{2}\right)$ & $89,3(77,8 ; 100,7)$ & $75,0(57,7 ; 92,3)$ & 80,6 & 85,7 & 82,7 \\
\hline Excesso de peso $\left(>27 \mathrm{~kg} / \mathrm{m}^{2}\right)$ & $60,7(42,6 ; 78,8)$ & $87,5(74,3 ; 100,7)$ & 85,0 & 65,6 & 73,1 \\
\hline Obesidade $\left(\geq 30 \mathrm{~kg} / \mathrm{m}^{2}\right)$ & $25,0(9,0 ; 41,0)$ & $100,0(100,0 ; 100,0)$ & 100,0 & 53,3 & 59,6 \\
\hline \multicolumn{6}{|l|}{$\mathrm{CC}^{\star}(\mathrm{cm})$} \\
\hline$\geq 90$ & $96,3(89,2 ; 103,4)$ & $50,0(30,0 ; 70,0)$ & 68,4 & 92,3 & 74,5 \\
\hline$\geq 94$ & $85,2(71,8 ; 98,6)$ & $58,3(38,6 ; 78,1)$ & 69,7 & 77,8 & 72,5 \\
\hline$\geq 102$ & $55,6(36,8 ; 74,3)$ & $91,7(80,6 ; 102,7)$ & 88,2 & 64,7 & 72,5 \\
\hline \multicolumn{6}{|l|}{ Feminino $(n=80)$} \\
\hline \multicolumn{6}{|l|}{ Categorias de IMC } \\
\hline Sobrepeso $\left(\geq 25 \mathrm{~kg} / \mathrm{m}^{2}\right)$ & $82,2(71,1 ; 93,4)$ & $77,1(63,2 ; 91,1)$ & 82,2 & 77,1 & 80,0 \\
\hline Excesso de peso $\left(>27 \mathrm{~kg} / \mathrm{m}^{2}\right)$ & $73,3(80,4 ; 86,3)$ & $85,7(74,1 ; 97,3)$ & 86,8 & 71,4 & 78,8 \\
\hline Obesidade $\left(\geq 30 \mathrm{~kg} / \mathrm{m}^{2}\right)$ & $53,3(38,8 ; 67,9)$ & $97,1(91,6 ; 102,7)$ & 96,0 & 61,8 & 72,5 \\
\hline \multicolumn{6}{|l|}{$\mathrm{CC}(\mathrm{cm})$} \\
\hline$\geq 80^{* *}$ & $97,8(93,5 ; 102,1)$ & $40,0(23,8 ; 56,2)$ & 67,7 & 93,3 & 72,5 \\
\hline$\geq 88$ & $84,4(73,9 ; 95,0)$ & $68,6(53,2 ; 84,0)$ & 77,6 & 77,4 & 77,5 \\
\hline
\end{tabular}

cesso de gordura corporal/obesidade em idosos de ambos os sexos. Essa é uma contribuição importante na área de estudos sobre antropometria, envelhecimento e morbidades, uma vez que existe controvérsia sobre a capacidade de predição desses indicadores antropométricos em idosos ${ }^{3,4}$.
Importantes modificações fisiológicas que ocorrem durante o processo de envelhecimento contribuem para as variações na composição corporal, não permitindo que um único ponto de corte de obesidade possa ser aplicado de maneira uniforme em diferentes faixas etárias e em diversas 
Tabela 3. Desempenho de pontos de corte de IMC e CC, obtidos por meio da curva ROC, para detecção de obesidade em idosos do sexo masculino e feminino, Goiânia, Brasil. $(\mathrm{n}=132)$

\begin{tabular}{|c|c|c|c|c|}
\hline & Ponto de Corte & $\begin{array}{c}\text { Sensibilidade } \\
\text { (IC95\%) }\end{array}$ & $\begin{array}{c}\text { Especificidade } \\
\text { (IC95\%) }\end{array}$ & $\begin{array}{c}\text { Acurácia } \\
(\%)\end{array}$ \\
\hline \multicolumn{5}{|c|}{ Masculino $(n=52)$} \\
\hline IMC & 25,0 & $89,3(77,8 ; 110,7)$ & $75,0(57,7 ; 92,3)$ & 82,7 \\
\hline $\mathrm{CC}^{*}$ & 98,8 & $81,5(66,8 ; 96,1)$ & $87,5(74,3 ; 100,7)$ & 84,3 \\
\hline \multicolumn{5}{|c|}{ Feminino $(n=80)$} \\
\hline IMC & 26,6 & $82,2(71,1 ; 93,4)$ & $85,7(74,1 ; 97,3)$ & 83,7 \\
\hline $\mathrm{CC}$ & 90,5 & $77,8(63,0 ; 88,1)$ & $74,3(59,8 ; 88,8)$ & 76,2 \\
\hline
\end{tabular}

${ }^{\star}$ Dados ausentes para 01 participante do sexo masculino. IMC: Índice de Massa Corporal; CC: Circunferência da Cintura.

populações, e é nesse sentido que este estudo traz subsídios e novas proposições.

Por outro lado, a análise de desempenho dos pontos de corte da CC existentes na literatura apresentaram acurácia inferior a 74\% e valores mais baixos de especificidade ou de sensibilida$\mathrm{de}^{4}$. O IMC $\geq 30 \mathrm{~kg} / \mathrm{m}^{2}$ também apresentou um fraco desempenho, com sensibilidade de $25 \%$ para homens e 53,3\% para mulheres, resultados coerentes com estudos de diferentes populações da Austrália, América no Norte, China e em metánalise de 25 estudos com 31.968 indivíduos ${ }^{3,6,24}$. $\mathrm{O}$ IMC $\geq 30 \mathrm{~kg} / \mathrm{m}^{2}$ é ainda recomendado por instituições internacionais como ponto de corte para obesidade em idosos de ambos os sexo, apesar das críticas em diferentes estudos ${ }^{14}$.

Pontos de corte altos de IMC, como o $30 \mathrm{~kg} /$ $\mathrm{m}^{2}$, não são apropriados para idosos, tendo em vista que falham em identificar aqueles com excesso de peso, devido à baixa sensibilidade. Como identificado neste e em outro ${ }^{25}$, valores de IMC de $27 \mathrm{~kg} / \mathrm{m}^{2}$ e $25 \mathrm{~kg} / \mathrm{m}^{2}$ são mais sensíveis e acurados. Isso provavelmente ocorre em função do aumento da gordura corporal, diminuição da massa muscular e óssea durante o processo de envelhecimento, sendo mais evidente em mulheres do que nos homens ${ }^{25}$; por isso, os pontos de corte de IMC e CC devem considerar essas diferenças. Por outro lado, a análise de dempenho dos valores mais baixos dos pontos de corte de IMC $\left(25 \mathrm{~kg} / \mathrm{m}^{2}\right.$ e $\left.27 \mathrm{~kg} / \mathrm{m}^{2}\right)$ apresentaram melhor o equilíbrio dos valores entre sensibilidade e especificidade e melhor a acurácia.

A obesidade central aferida pela circunferencia da cintura tem sido proposta como um dos melhores preditores antropométricos de gordura visceral e risco de doenças metabólicas ${ }^{14,26}$, tendo maior maior associação com mortalidade e mais fortemente correlacionada com fatores de risco do que o IMC ${ }^{8,26}$. No entanto, devido à variabilidade de composição corporal, estudos de desempenho da CC são necessários para a população idosa, uma vez que associa-se a condições negativas em saúde $7,8,27$. Assim, sendo necessária a definição de pontos de corte específicos sobre o excesso de gordura corporal para esta população ${ }^{8,28}$.

Com relação aos pontos de corte da CC analisados neste estudo, verificou-se que os pontos de corte $\geq 90 \mathrm{~cm}$ para homens e $\geq 80 \mathrm{~cm}$ para $\mathrm{mu}$ lheres (IDF) ${ }^{29}$ e CC $\geq 102 \mathrm{~cm}$ para homens e $\geq 88$ $\mathrm{cm}$ para mulheres (NCEP) apresentaram melhor acurácia. Por outro lado, os pontos de corte $\geq 94$ $\mathrm{cm}$ para homens e $\geq 80 \mathrm{~cm}$ para mulheres (WHO) apresentaram baixa especificidade em ambos os sexos e, como consequência, maior percentual de falso-positivos. Portanto, mesmo considerando os pontos de corte acima de $90 \mathrm{~cm} \mathrm{e} 80 \mathrm{~cm}$ para homens e mulheres respectivamente (IDF), o mesmo não deve ser utilizado nas mulheres ${ }^{29}$. Além disso, estudo com 3.435 adultos tunisianos demonstrou que o melhor ponto de corte de CC foi $85 \mathrm{~cm}$ nos homens e $79 \mathrm{~cm}$ nas mulheres com elevada especificidade e sensibilidade ${ }^{30}$.

Estudos em população asiática ${ }^{31}$, coreana e chinesa $^{4,18}$ demonstraram pontos de corte diferentes, o que reforça a importância do presente estudo com a sugestão de pontos de corte específicos para a população idosa brasileira.

Nesse sentido, estudos brasileiros avaliaram parâmetros antropométricos (CC e/ou IMC) em idosos na predição da obesidade $\mathrm{e}^{5,6,25}$ e de risco cardiovascular ${ }^{5}$. Observa-se importante variação entre os pontos de corte sugeridos de IMC e CC tendo diferentes níveis de sensibilidade e especificidade ${ }^{5}$. Os pontos de corte do presente estudo se assemelham mais aos sugeridos por Barbosa et al. ${ }^{25}$, que de forma geral apresentou melhor sensibilidade, especificidade e acurácia nos pontos 


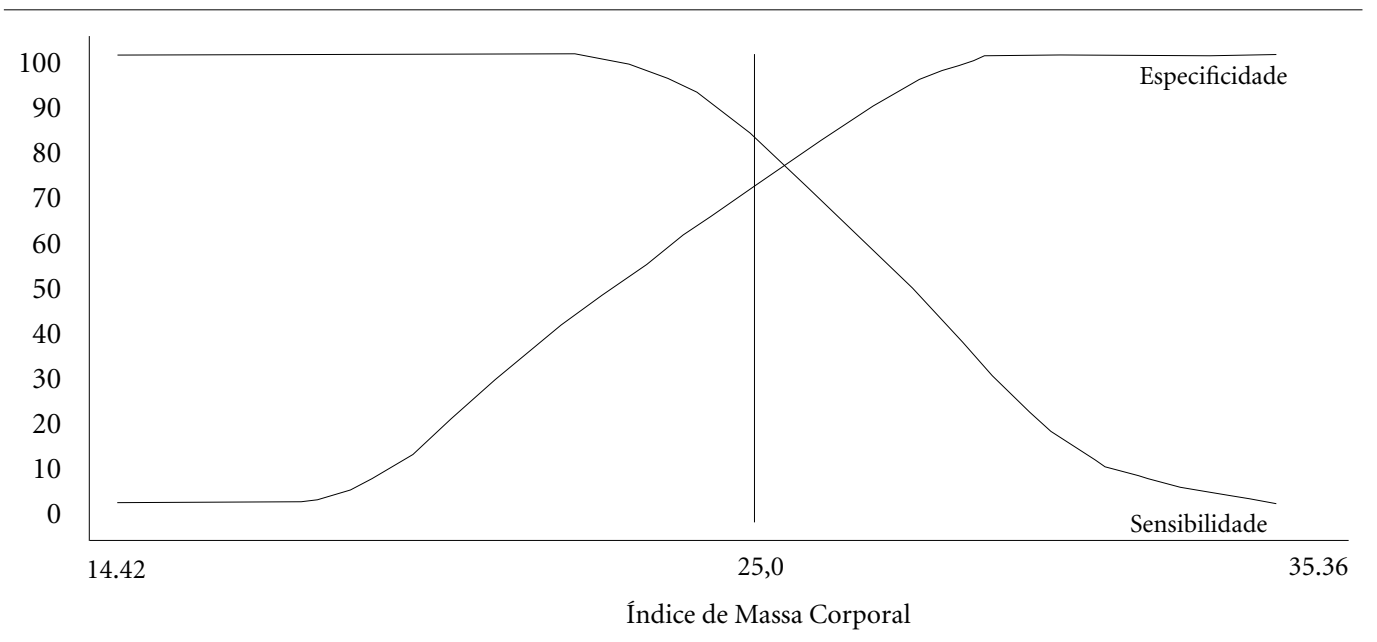

Área sob a curva ROC: 0,8817 (0,79199; 0,97140)

Figura 1. Curva ROC para identificação de pontos de corte de Índice de massa corporal para diagnosticar excesso de gordura corporal em idosos do sexo masculino e feminino. Goiânia, Brasil. ( $\mathrm{n}=132$ )

Figura 1a. Sexo Masculino.

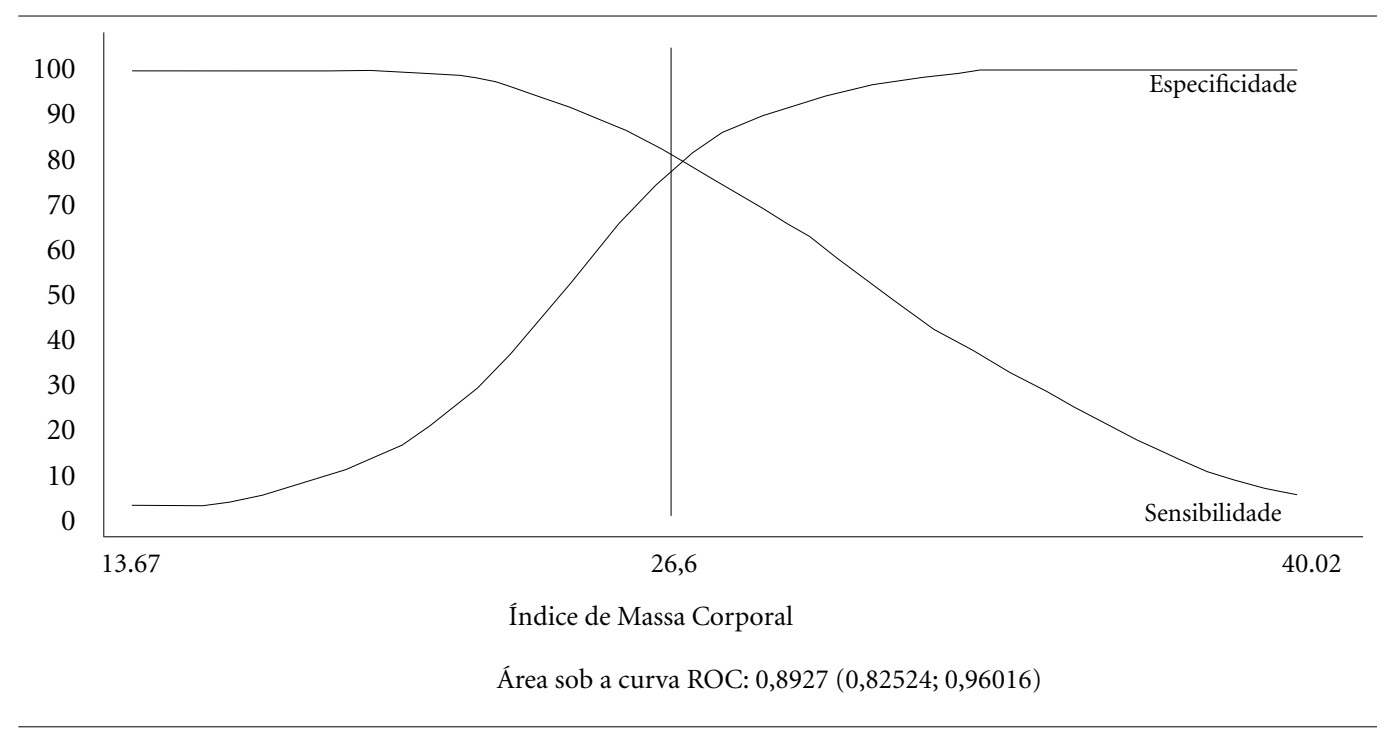

Figura 1b. Sexo Feminino.

de corte sugeridos. Diferenças encontradas entre estudos internacionais e nacionais bem como entre diferentes regiões do mesmo país, se deve possivelmente aos diferentes desfechos, métodos de análise e resultados apresentados, além da heterogeneidade populacional.

Os pontos de corte sugeridos tem potencial para utilização em outras populações de idosos do Brasil e da América do Sul. Essa recomenda- ção é particularmente importante, pois os pontos de corte que são aplicados nesses países, foram determinados para populações diversas e distantes do seu real perfil ${ }^{21,29}$. Os pontos de corte do IMC utilizados atualmente para diagnóstico da obesidade, têm alta especificidade, mas sensibilidade menor que $50 \%$ para diagnóstico de excesso de gordura corporal. Os nossos resultados reforçam os de publicações internacionais de que os 


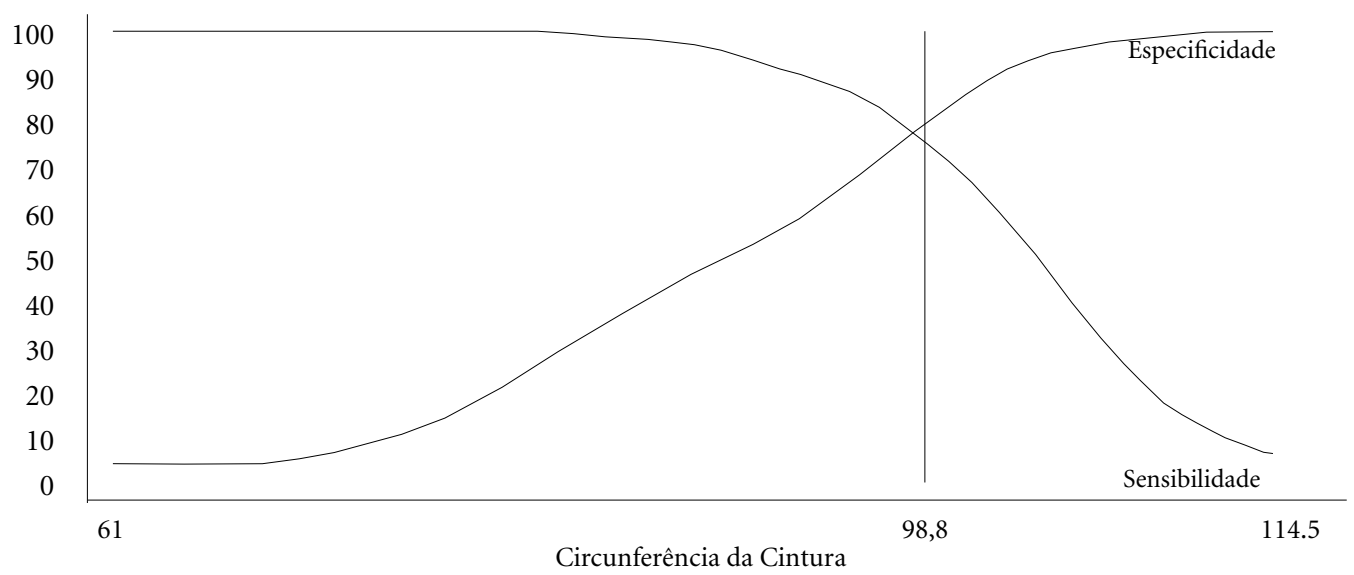

Área sob a curva ROC: $0,8812(0,78779 ; 0,97455)$

Figura 2. Curva ROC para identificação de pontos de corte de Circunferência da cintura para diagnosticar excesso de gordura corporal em idosos do sexo masculino e feminino. Goiânia, Brasil. ( $\mathrm{n}=132)$

Figura 2a. Sexo Masculino.

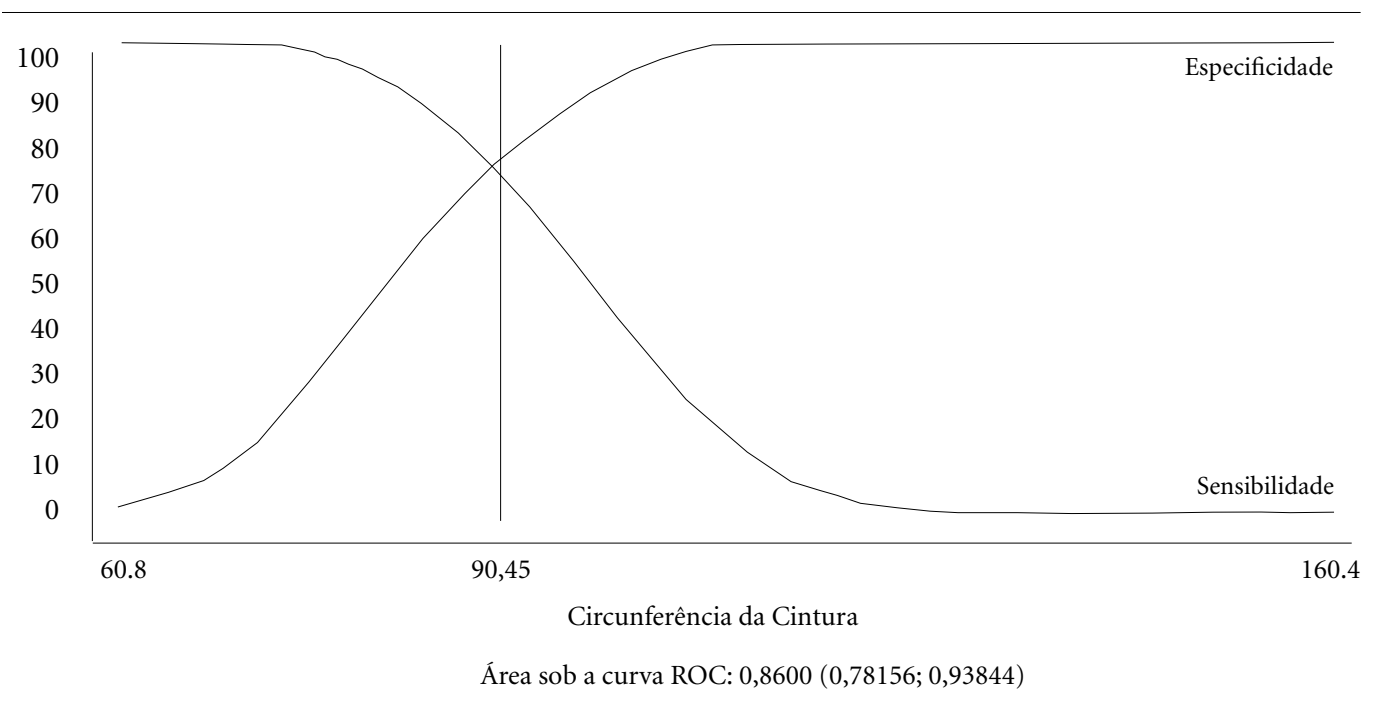

Figura 2b. Sexo Feminino.

pontos de corte existentes não consideram o ganho de gordura corporal decorrente do aumento da idade.

Assim, os resultados da presente pesquisa ajudam a determinar pontos de corte de CC mais sensíveis e específicos a fim de aprimorar o diagnóstico de obesidade com medidas antropométricas simples entre idosos brasileiros e, com isso, contribuir para o desenvolvimento de estratégias de tratamento e prevenção de outras morbidades associadas.

Como limitação deste estudo, poderia ser citado o número de idosos analisados, porém como se trata de um estudo para analisar acurácia e validação de medidas antropométricas, esse número é suficiente conforme cálculo realizado $a$ priori. Vale ressaltar neste estudo o padrão ouro utilizado, a densitometria corporal total, técnica 
com alta precisão no diagnóstico de composição corporal o que valida ainda mais a comparação das medidas antropométricas e os resultados apresentados.

Portanto, recomenda-se o uso dos pontos de corte específicos para idosos propostos neste estudo, sendo de $25 \mathrm{~kg} / \mathrm{m}^{2}$ e $98,8 \mathrm{~cm}$ para o sexo masculino e $26,6 \mathrm{~kg} / \mathrm{m}^{2}$ e $90,5 \mathrm{~cm}$ no sexo feminino, a fim de melhor predizer o excesso de gordura corporal/obesidade. A aplicação desses pontos de corte mais acurados de IMC e CC nos serviços de saúde e em programas de saúde da família podem aprimorar os diagnósticos de obesidade em idosos da comunidade. A importância da definição de pontos de corte se destacam por sua simplicidade operacional e boa acurácia sendo de grande utilidade nos serviços de atenção à saúde, além de possibilitar o conhecimento da situação de grupos populacionais específicos quando empregados na pesquisa epidemiológica.

\section{Colaboradores}

EA Silveira - Elaboração da concepção do estudo, coleta e análise de dados, revisão de literatura, escrita do manuscrito e revisão final. V Pagotto - Coleta de dados, revisão da análise estatística, escrita do manuscrito e revisão final. LS Barbosa - Elaboração da concepção do estudo, coleta e análise de dados, escrita do manuscrito e revisão final. C Oliveira - Planejamento e revisão da análise estatística, revisão final do texto do artigo com contribuições, elaboração da versão em inglês. GG Pena - revisão de literatura, escrita do manuscrito e revisão final. G Velasquez-Melendez - revisão da análise estatística, escrita do manuscrito e revisão final.

\section{Agradecimentos}

G Velasquez-Melendez e LS Barbosa receberam bolsa de CNPq.

Pesquisa financiada pelo CNPq. 


\section{Referências}

1. Swinburn BA, Sacks G, Hall KD, McPherson K, Finegood DT, Moodie ML, Gortmaker SL. The global obesity pandemic: shaped by global drivers and local environments. Lancet 2011; 378(9793):804-814.

2. Agborsangaya $\mathrm{CB}$, Ngwakongnwi E, Lahtinen $\mathrm{M}$, Cooke T, Johnson JA. Multimorbidity prevalence in the general population: the role of obesity in chronic disease clustering. BMC Public Health 2013; 13:1161.

3. Okorodudu DO, Jumean MF, Montori VM, Romero-Corral A, Somers VK, Erwin PJ, Lopez-Jimenez F. Diagnostic performance of body mass index to identify obesity as defined by body adiposity: A systematic review and meta-analysis. Int J Obes 2010; 34(5):791799.

4. Hou XG, Wang C, Ma ZQ, Yang WF, Wang JX, Li CQ, Wang YL, Liu SM, Hu XP, Zhang XP, Jiang M, Wang WQ, Ning G, Zheng HZ, Ma AX, Sun Y, Song J, Lin P, Liang K, Liu FQ, Li WJ, Xiao J, Gong L, Wang MJ, Liu JD, Yan F, Yang JP, Wang LS, Tian M, Zhao RX, Jiang L, Chen L. Optimal waist circumference cut-off values for identifying metabolic risk factors in middle-aged and elderly subjects in Shandong Province of China. Biomed Env Sci 2014; 27(5):353-359.

5. Martins MV, Queiroz Ribeiro A, Martinho KO, Silva Franco F, Souza JD, Morais KBD, Gonçalves Leite IC Araújo Tinôco AL. Anthropometric indicators of obesity as predictors of cardiovascular risk in the elderly. Nutr Hosp 2015; 31(6):2583-2589.

6. Vasconcelos FAG, Cordeiro BA, Rech CR, Petroski EL. Sensitivity and specificity of the body mass index for the diagnosis of overweight/obesity in elderly. Cad Saude Publica 2010; 26(8):1519-1527.

7. Gharipour M, Sadeghi M, Dianatkhah M, Bidmeshgi S, Ahmadi A, Tahri M, Sarrafzadegan N. The cut-off values of anthropometric indices for identifying subjects at risk for metabolic syndrome in iranian elderly men. J Obes 2014; 2014:907149.

8. de Hollander EL, Bemelmans WJ, Boshuizen HC, Friedrich N, Wallaschofski $\mathrm{H}$, Guallar-Castillón P, Walter S, Zillikens MC, Rosengren A, Lissner L, Bassett JK, Giles GG, Orsini N, Heim N, Visser M, de Groot LC WC elderly collaborators. The association between waist circumference and risk of mortality considering body mass index in 65- to 74-year-olds: A meta-analysis of 29 cohorts involving more than 58000 elderly persons. Int J Epidemiol 2012; 41(3):805-817.

9. Pagotto V, Nakatani AYK, Silveira EA. Fatores associados à autoavaliação de saúde ruim em idosos usuários do Sistema Único de Saúde. Cad Saude Publica 2011; 27(8):1593-1602.

10. Pagotto V, Silveira EA. Methods, Diagnostic Criteria, Cutoff Points, and Prevalence of Sarcopenia among Older People. Sci World J 2014; 2014:231312.

11. Silva RRV, Silveira MF, Pena GG, Martins TCR, Pinheiro UB, Monteiro Júnior RS, Santos SHS, Paula AMB, Rodrigues Neto JF, Guimarães ALS. Genetic variation in the promoter region of the TNF rs1800629 gene is not associated with adiposity index, but AA genotype is more likely to have low cellular membrane integrity. Meta Gene 2017; 13:85-91.

12. Browner W, Newman T, Cummings S, Huley S. Designing Clinical Research: an epidemiologic approach. Philadelphia: LWW; 2001.
13. Gordon C, Chumlea WRT. Stature, recumbent length, and weight. In: Anthropometric Standardization Reference Manual. Champaign: Human Kinetics Books; 1988. p. 3-8.

14. World Heatlh Organization (WHO). Physical status: the use and interpretation of anthropometry. Report of a WHO Expert Committee. Geneva: WHO; 1995. [World Health Organization technical report series 854]

15. Nutrition Screening Iniciative (NSI). Nutrition interventions manual for professionals caring for older Americans. Washington: NSI; 1992.

16. Silveira EA, Kac G, Barbosa LS. Prevalência e fatores associados à obesidade em idosos residentes em Pelotas, Rio Grande do Sul, Brasil: classificação da obesidade segundo dois pontos de corte do índice de massa corporal. Cad Saude Publica 2009; 25(7):1569-1577.

17. Lohman TG, Roche AF, Martorell R. Anthropometric Standardization Reference Manual. Champaign: $\mathrm{Hu}-$ man Kinetics Books; 1988

18. Baik I. Optimal Cutoff Points of Waist Circumference for the Criteria of Abdominal Obesity. Circ J 2009, 73(11):2068-2075.

19. Rotterdam ESHRE/ASRM-Sponsored PCOS consensus workshop group. Revised 2003 consensus on diagnostic criteria and long-term health risks related to polycystic ovary syndrome (PCOS). Hum Reprod 2004; 19(1):41-47.

20. National Institutes of Health (NIH). Clinical Guidelines on the Identification, Evaluation, and Treatment of Overweight and Obesity in Adults-The Evidence Report. Obesity research. Bethesda: NIH; 1988.

21. International Diabetes Federation (IDF). The IDF consensus worldwide definition of the metabolic syndrome. Brussels: IDF; 2005.

22. Kyle UG, Genton L, Slosman DO, Pichard C. Fat-free and fat mass percentiles in 5225 healthy subjects aged 15 to 98 years. Nutrition 2001; 17(7-8):534-541.

23. Habicht J-P. Estandarizacion de métodos epidemiológicos cuantitativos sobre el terreno. Bolletin de la Oficina Sanitaria Panamericana 1974; 76(5):374-384.

24. Romero-Corral A, Somers V, Sierra-Johnson J, Thomas RJ, Collazo-Clavell ML, Korinek J, Allison TG, Batsis JA, Sert-Kuniyoshi FH, Lopez-Jimenez F. Accuracy of Body Mass Index to Diagnose Obesity In the US Adult Population. Int J Obes 2010; 32(6):959-966.

25. Barbosa MH, Bolina AF, Luiz RB, Oliveira KF, Virtuoso Júnior JS, Rodrigues RA, Silva LC, Cunha DF, De Mattia AL, Barichello E. Body mass index as discriminator of the lean mass deficit and excess body fat in institutionalized elderly people. Geriatr Nurs 2015 36(3):202-206

26. Zhu S, Wang Z, Heshka S, Heo M, Faith MS, Heymsfield SB. Waist circumference and obesity-associated risk factors among whites in the third National Health and Nutrition Examination. Am J Clin Nutr 2002, 76(4):743-749.

27. Fauziana R, Jeyagurunathan A, Abdin E, Vaingankar J, Sagayadevan V, Shafie S, Sambasivam R, Chong SA, Subramaniam M. Body mass index, waist-hip ratio and risk of chronic medical condition in the elderly population: Results from the Well-being of the Singapore Elderly (WiSE) Study. BMC Geriatr 2016; 16:125. 
28. Chen Y-M, Ho SC, Lam SSH, Chan SSG. Validity of body mass index and waist circumference in the classification of obesity as compared to percent body fat in Chinese middle-aged women. Int J Obes 2006; 30(6):918-925.

29. Alberti KG, Eckel RH, Grundy SM, Zimmet PZ, Cleeman JI, Donato KA, Fruchart JC, James WP, Loria CM, Smith SC Jr; International Diabetes Federation Task Force on Epidemiology and Prevention; Hational Heart, Lung, and Blood Institute; American Heart Association; World Heart Federation; International Atherosclerosis Society; International Association for the Study of Obesity. Harmonizing the metabolic syndrome: A joint interim statement of the international diabetes federation task force on epidemiology and prevention; National heart, lung, and blood institute; American heart association; World heart federation; International atherosclerosis society; And international association for the study of obesity. Circulation 2009; 120(16):1640-1645.

30. Bouguerra R, Alberti H, Smida H, Salem LB, Rayana CB, El Atti J, Achour A, Gaigi S, Slama CB, Zouari B, Alberti KG. Waist circumference cut-off points for identification of abdominal obesity among the tunisian adult population. Diabetes Obes Metab 2006; 9(6):859-868.

31. Aye M, Sazali M. Waist circumference and BMI cutoff points to predict risk factors for metabolic syndrome among outpatients in a district hospital. Singapore Med J 2012; 53(8):545-550.

Artigo apresentado em 29/11/2017

Aprovado em 19/07/2018

Versão final apresentada em 21/07/2018 